\title{
Identification of objective pathological prognostic determinants and models of prognosis in Dukes' B colon cancer
}

\author{
V C Petersen, K J Baxter, S B Love, N A Shepherd
}

See end of article for authors' affiliations

......................

Correspondence to: Professor N A Shepherd, Department of Histopathology, Gloucestershire Royal Hospital, Great Western Road, Gloucester GLI 3NN, UK; neil.shepherd@ gloucr-tr.swest.nhs.uk

Accepted for publication 20 November 2001

\begin{abstract}
Background and aims: There is a need for objective easily determined pathological prognostic parameters in Dukes' B colon carcinoma to allow selection of such patients for further treatment as the role of adjuvant chemotherapy for these patients remains unclear. This study was initiated to assess the influence of pathological factors on prognosis in an unselected prospective series of Dukes' B colonic cancer.

Methods: The Gloucester Colorectal Cancer study, established in 1988, recruited more than 1000 cases. Meticulous pathological assessment of the 268 Dukes' B colonic cancer resections in this series included evaluation of all pathological factors that could influence staging and prognosis. All patients entered a comprehensive follow up system.

Results: Four pathologically determined factors-peritoneal involvement, venous spread (both submucosal and extramural), spread to involve a surgical margin, and perforation through the tumour-were independent prognostic factors in multivariate analysis. Combining these four factors into a simple cumulative scoring system generated clinically useful prognostic groups.

Conclusions: The cumulative prognostic index allows apportionment of patients with Dukes' B colon cancer into defined prognostic groups, which in turn could allow more objective selection of patients for adjuvant therapy, especially as part of clinical trials.
\end{abstract}

$\mathrm{T}$ he prognosis of patients who have carcinoma of the colon or rectum is dependent on several factors: clinical, pathological, and biological. Among the pathological factors, penetration of the bowel wall and local lymph node involvement are the two most powerful prognostic indicators: these factors constitute the Dukes' staging system which remains the most important determinant of the decision to institute postoperative chemotherapy in both colonic and rectal cancer. ${ }^{1}$ The efficacy of adjuvant chemotherapy in Dukes' C (lymph node positive) cancer, colonic and rectal, is largely undisputed and has been shown to produce a reduction in recurrence and mortality, to increase disease free survival, and to be cost effective..$^{2-7}$ However, the role of adjuvant therapy in Dukes' B carcinoma is still debated and has yet to be clarified. There have been conflicting results from large trials. ${ }^{34-13}$ Accurate patient selection is a critical part of the decision to institute adjuvant treatment. For instance, in the rectum, the extent of mesorectal spread and involvement of the deep (circumferential, radial, mesorectal) margin are important determinants of local recurrence and this prognostic determinant is currently used to select patients for adjuvant therapy, particularly radiotherapy. ${ }^{14-16}$ This is also the subject of the current MRC trial, CRO7. In the colon, unlike rectal cancer, radiotherapy is largely unsuitable for carcinoma and its efficacy unproven. Thus chemotherapy, usually systemic but also potentially intraperitoneal, remains the mainstay of adjuvant therapy in colonic carcinoma. ${ }^{2}{ }^{17}$

In most series, between $40 \%$ and $50 \%$ of colonic carcinomas are Dukes' B stage. ${ }^{18}$ To subject all of these patients to chemotherapy may be inappropriate and costly. ${ }^{79}$ Dukes' $^{\prime} \mathrm{B}$ cancer represents a very wide spectrum of disease from very early penetration through the bowel wall, with a prognosis approaching that of Dukes' A cancer, to aggressive and extensive tumours with extramural venous spread and involvement of the serosa, surgical margins, or adjacent organs. There is therefore an increasing need for accurate stratification of
Dukes' B colonic cancers to identify those with higher rates of locoregional recurrence and subsequent relapse and to identify those for whom adjuvant chemotherapy may be of greater benefit.

\section{MATERIALS AND METHODS}

The Gloucester Colorectal Cancer Study was instituted in 1988. A total of 1050 patients, 673 with colonic cancer and 377 with rectal cancer, were recruited between August 1988 and September 1996. Two hundred and sixty eight (39.8\%) of the colonic cancers were Dukes' B stage, representing a prospective, continuous, unselected cohort of patients who underwent a primary resection in Gloucester between these dates. The Gloucestershire Local Research Ethics Committee, under reference $01 / 21 \mathrm{G}$, approved the study.

Curative and palliative cases were included although cases in which resection was performed for synchronous carcinoma, metachronous carcinoma, and carcinoma arising in ulcerative colitis and familial adenomatous polyposis were excluded. Cases were considered curative if the surgeon and/or the pathologist judged that all tumour had apparently been removed by the end of the surgical procedure. Cases deemed palliative included those with metastatic disease, particularly to the liver and/or lung, local tumour spread beyond the surgical margin, and tumour perforation. As some of these factors (especially the latter two, both of which required histological confirmation in this study) are important prognostic factors in Dukes' B colonic cancer, the curative/palliative status was included in the analysis but distinction between these categories is not regarded as an important aspect of this study.

In each case, one pathologist (NAS) carried out the pathological analysis of each resection specimen in a standardised

Abbreviations: $\mathrm{Pl}$, prognostic index. 
Table 1 Categorisation of pathological prognostic factors in Dukes' B colon cancer

\begin{tabular}{llr}
\hline Factor & Category & No of patients $(\%)$ \\
\hline Extent of spread beyond muscularis propria & Slight $(<2 \mathrm{~mm})$ & $72(26.9)$ \\
& Moderate $(3-5 \mathrm{~mm})$ & $110(41.0)$ \\
& Extensive $(>5 \mathrm{~mm})$ & $86(32.1)$ \\
Peritoneal involvement & Absent & $52(19.4)$ \\
& Inflammatory & $105(39.2)$ \\
& Present & $61(22.8)$ \\
& With ulceration & $50(18.7)$ \\
& Not evident & $153(57.1)$ \\
& Submucosal & $24(9.0)$ \\
& Extramural & $91(34.0)$ \\
Margin involvement & Not involved & $232(86.6)$ \\
& Inflammatory & $28(10.5)$ \\
Tumour perforation & Present & $8(3.0)$ \\
& Absent & $257(95.9)$ \\
Tumour differentiation & Present & $11(4.1)$ \\
& Well & $56(20.9)$ \\
Adjacent organ involvement & Moderate & $157(58.6)$ \\
& Poor & $55(20.5)$ \\
& Absent & $238(88.8)$ \\
& Present & $30(11.2)$ \\
\hline
\end{tabular}

meticulous manner. This involved harvesting of all lymph nodes (mean lymph node harvest 21.3) and comprehensive sampling of the tumour for histology (mean number of tumour blocks 5.7). The latter allowed a comprehensive analysis of potential extramural venous spread, as previously described..$^{1820}$ At least two blocks were taken from each case where tumour was closest to the peritoneal surface. ${ }^{18}$ Two blocks were also taken from the area where tumour was closest to any surgical margin, whether retroperitoneal (for instance in the caecum) or mesocolic.

Microscopic assessment included the recording, using standard methodology, of tumour type, tumour grade, and intratumoral fibrosis. ${ }^{18}{ }^{21-24}$ Venous spread was assessed histologically in the three groups (table 1) using conventional methodology. ${ }^{20}$ Peritoneal involvement was divided into four groups, as previously described (table 1). ${ }^{125}$ The extent of spread was determined histologically as a measurement from the outer border of the longitudinal muscle layer to the most distant point of tumour spread and divided into three groups, as previously described (table 1). ${ }^{18}{ }^{25}$ Involvement of a surgical margin was assessed histologically according to established criteria for rectal cancer: thus if the tumour was within $1 \mathrm{~mm}$ of a margin, this was considered involved. ${ }^{14}$ A third category (table 1) was introduced to allow identification of a relatively common feature in colonic cancer where acute inflammation and suppuration are present at a margin with tumour in continuity, through the suppuration, with the margin although the tumour itself does not actually involve the margin histologically. Perforation was only deemed to be present if there was histological evidence of perforation through the tumour. Adjacent organ involvement was also only deemed to be present if confirmed by histology.

While a meticulous technique to harvest all lymph nodes was undertaken, any involvement of nodes, and thus Dukes' C stage, precluded inclusion of the relevant case in this study. Jass parameters, ${ }^{26}$ lymphocytic infiltrate, and quality of advancing margin were not included in this study because they have been deemed to be too subjective in several studies and are no longer recommended for routine usage in standard UK and international reporting protocols.

Each patient was regularly followed up with surgical outpatient assessment (for a minimum of five years) and close collaboration with general practitioners. All clinical, pathological, follow up, and survival data were stored on a computer database and regularly updated by a research officer (KJB). Survival time was calculated from the date of surgery to the date of death or last follow up, with times censored for patients dying of causes not related to colonic cancer and those still alive. Only cancer related deaths were analysed as events. Cause of death was established by autopsy or, in the absence of a post mortem examination, the judgement was made on careful appraisal of the clinical course of the patient. If there was any doubt concerning the cause of death, survival time was censored at the date of death. Data concerning any adjuvant therapy were incorporated in the database and subjected to analysis as part of this study.

The log rank test and Cox multivariate regression analysis were used to build a traditional prognostic model. ${ }^{27}$ Factors found to have a significance less than 0.1 in the log rank test were entered into a stepwise Cox regression model to give a final model of independent prognostic factors. This model was checked for the proportional hazard assumption, for the effect of tied failed survival times, for outliers, for leverage points, and for overall model fit. Tumour perforation was a substantial risk factor for prognosis in the first post surgical year. Hence it was modelled as a time varying factor, increasing the risk for the first year only.

The model was internally validated using bootstrapping. ${ }^{28} \mathrm{~A}$ bootstrap of 100 samples of 268 patients was performed using backwards elimination stepwise Cox analysis of the factors found to have a significance of less than 0.1 in the log rank test. A high risk group was selected using the prognostic index from this Cox model. ${ }^{27}$

\section{RESULTS}

Of the 268 patients, there were 143 males and 125 females with a mean age of 72 years (range 39-92). The distribution of the tumours was as follows: $44(16 \%)$ in the caecum, $56(21 \%)$ in the ascending colon and hepatic flexure, $42(16 \%)$ in the transverse colon and splenic flexure, $17(6 \%)$ in the descending colon, and $109(41 \%)$ in the sigmoid colon. A total of 239 $(89 \%)$ operations were deemed curative and 29 (11\%) palliative according to our criteria. Five patients were lost to follow up at 48, 24, 14, 10, and 3 months and a further 39 patients have still to reach five years of follow up. At the time of analysis, there had been 63 cancer related deaths, a median follow up of 65 months, and a five year survival rate of $76 \%$ (95\% confidence interval (CI) $70-81 \%$ ). Fifty seven patients in the series had died from non-cancer related deaths.

Of the 268 patients, 21 had radiotherapy or chemotherapy as well as surgical intervention (including only 3/29 palliative surgery patients and none of 11 patients with tumour 
Table 2 Univariate analysis of pathological prognostic factors in Dukes' B colon cancer

\begin{tabular}{|c|c|c|c|c|}
\hline Factor & Category & $\begin{array}{l}\% 5 \text { year survival } \\
(95 \% \mathrm{Cl})\end{array}$ & $\begin{array}{l}\text { Log rank } \chi^{2} \\
\text { (df) }\end{array}$ & $\begin{array}{l}\text { Log rank } \\
\text { p value }\end{array}$ \\
\hline \multirow[t]{3}{*}{ Extent of spread } & Slight & 89.3 (78.7-94.7) & $15.00(2)$ & 0.0006 \\
\hline & Moderate & $76.9(67.2-84.1)$ & & \\
\hline & Extensive & $61.5(48.7-72.0)$ & & \\
\hline \multirow[t]{4}{*}{ Peritoneal involvement } & Absent & 84.3 (69.5-92.3) & $29.53(3)$ & $<0.0001$ \\
\hline & Inflammatory & $88.4(80.1-93.4)$ & & \\
\hline & Present & $67.0(52.6-78.0)$ & & \\
\hline & With ulceration & $50.9(35.3-64.6)$ & & \\
\hline \multirow[t]{2}{*}{ Peritoneal involvement } & Absent/inflammatory & $87.1(80.2-91.7)$ & $21.73(1)$ & $<0.0001$ \\
\hline & Presenttulceration & $59.9(49.4-68.6)$ & & \\
\hline \multirow[t]{3}{*}{ Venous invasion } & Not evident & $83.7(76.2-89.0)$ & $16.05(2)$ & 0.0003 \\
\hline & Submucosal & $73.0(49.4-87.0)$ & & \\
\hline & Extramural & $61.6(49.6-71.5)$ & & \\
\hline \multirow[t]{2}{*}{ Venous invasion } & Not evident & 83.7 (76.2-89.0) & $15.23(1)$ & 0.0001 \\
\hline & Submucosal and/or extramural & 64.1 (53.7-72.8) & & \\
\hline \multirow[t]{3}{*}{ Margin involvement } & Absent & 78.7 (72.3-83.7) & $7.62(2)$ & 0.02 \\
\hline & Inflammatory & $57.1(35.8-73.6)$ & & \\
\hline & Present & $\begin{array}{l}\text { Longest survivor FU } \\
\text { only } 47 \text { months }\end{array}$ & & \\
\hline \multirow[t]{2}{*}{ Margin involvement } & Absent & 78.7 (72.3-83.7) & $7.31(1)$ & 0.007 \\
\hline & Inflamed or present & $55.3(35.8-73.6)$ & & \\
\hline \multirow[t]{2}{*}{ Tumour perforation } & Absent & $76.6(70.5-81.7)$ & $9.27(1)$ & 0.0023 \\
\hline & Present & $46.8(14.8-73.9)$ & & \\
\hline \multirow{2}{*}{$\begin{array}{l}\text { Adjacent organ } \\
\text { involvement }\end{array}$} & Absent & $78.4(72.1-83.4)$ & 7.08 (1) & 0.008 \\
\hline & Present & $51.7(29.9-69.7)$ & & \\
\hline
\end{tabular}

Table 3 Multivariate analysis of pathological prognostic factors in Dukes' B colon cancer

\begin{tabular}{lllcc}
\hline Factor & Comparison & Hazard ratio (95\% CI) & Coefficient & $Z$ \\
\hline Peritoneal involvement & Absent $v$ present & $2.88(1.69-4.90)$ & 1.06 & 3.906 \\
Venous invasion & Not evident $v$ present & $2.70(1.61-4.53)$ & 0.99 & 3.754 \\
Margin involvement & Absent $v$ present & $2.61(1.42-4.79)$ & 0.96 & 3.089 \\
Tumour perforation & Absent $v$ present & $9.43(3.28-27.05)$ & 2.24 & 0.0001 \\
\hline
\end{tabular}

perforation). There were nine cancer related deaths in these patients treated with adjuvant therapy. Adjustment for, or omission of, these patients had no significant effect on the results.

Log rank analysis identified six factors, with $\mathrm{p}<0.1$, shown in table 2. Age, sex, site, differentiation, and type were not found to be statistically significant prognostic factors. The final Cox regression model, identifying four independent prognostic factors, is shown in table 3. In our data, submucosal and extramural venous invasion showed similar prognostic significance and have therefore been combined. Similarly, the inflamed margin conferred a similar adverse prognosis to unequivocal involvement of the margin and these two categories have also been combined in the analysis.

In the bootstrap analysis of 100 samples of 268 patients, the four variable prognostic model was selected 44 times and at least three of the variables were selected 84 times. Thus the prognostic model showed a high degree of stability. The coefficients for the prognostic index (PI) are given in table 3, but can be illustrated by the simplified equation:

$\mathrm{PI}=1$ (if peritoneal involvement \pm ulceration)

+1 (if extramural or submucosal venous spread)

+1 (if margin involved or inflamed)

+2 (if perforation through tumour).

Hence PI can have values of 0 to 5 and the five year survival of these groups is given in table 4 (with categories 3, 4, and 5 combined due to small numbers). From these data, patients can be divided into a low risk group of those with a PI of 0 or l, with a five year survival of $85.7 \%$ (95\% CI $79.4-90.2 \%$ ), and a high risk group of those with a PI of 2 or more with a five year survival of $49.8 \%$ (95\% CI 37.0 - 61.3). A Kaplan-Meier curve of these low and high risk groupings is demonstrated in fig 1 .

Table 4 Prognostic index (PI) scoring with survival times

\begin{tabular}{lccc}
\hline PI score & Total patients & $\begin{array}{l}\text { Patients dying from } \\
\text { cancer }\end{array}$ & 5 year survival $(95 \% \mathrm{Cl})$ \\
\hline 0 & 82 & 6 & $94.2 \%(85.0-97.8)$ \\
1 & 109 & 21 & $79.5 \%(69.9-86.3)$ \\
2 & 63 & 28 & $54.3 \%(40.3-66.3)$ \\
$\geqslant 3$ & 14 & 8 & $30.4 \%(7.8-57.4)$ \\
Total & 268 & 63 & $76.1 \%(70.0-81.0)$ \\
\hline
\end{tabular}




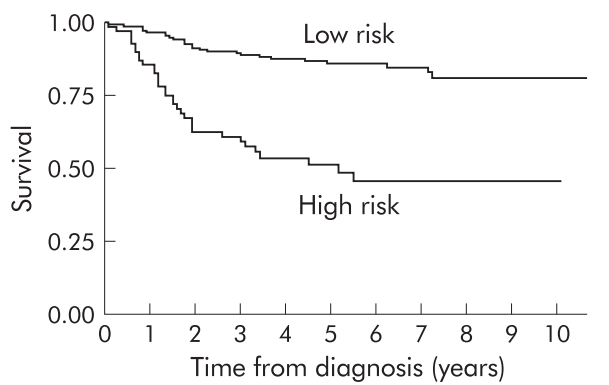

Figure 1 Kaplan-Meier survival graph of low and high risk Dukes' B colonic cancer.

\section{DISCUSSION}

With meticulous pathological examination of the resection specimen, the staging of colonic cancers, according to Dukes' classification, is relatively simple and reproducible. Dukes' B colonic tumours account for a large number of all colonic cancers (in this series 40\%), particularly because Dukes' A colonic carcinomas are rare compared with the rectum. The pathological features and clinical behaviour of Dukes' B colonic cancer are highly variable ${ }^{29}$ and there is a need to identify easily determined factors that may enable selection of patients by prognosis. This study suggests that four such factors can be easily demonstrated by routine pathological methods and, accordingly, are objective and we believe readily reproducible.

In previous studies, including all Dukes' stages, extramural venous spread has been shown to be of prognostic value $^{18} 2022$ and in this series restricted to Dukes' B colonic cancer patients, extramural venous spread was a powerful independent prognostic factor. Furthermore, submucosal venous spread showed adverse prognostic significance in this study. This is an important finding because this feature loses prognostic significance when all stages are combined, in rectal cancer at least. ${ }^{20}$ The significance of submucosal venous spread, in terms of ultimate prognosis, approaches that of extramural venous involvement and the two can be effectively combined to provide a robust and simple prognostic classification.

Local peritoneal involvement is a parameter which we have previously demonstrated to show powerful independent prognostic significance in colonic cancer ${ }^{18}$ and less powerful prognostic significance in rectal cancer. ${ }^{25}$ We believe that its powerful independent prognostic significance in colonic cancer is not only related to its ability to predict intraperitoneal metastasis but also because it identifies a patient group with local spread significantly beyond the bowel wall. ${ }^{1825}$ As Dukes' A cases are relatively unusual in colonic cancer, compared with rectal cancer, spread beyond the muscularis propria is almost universal in colonic cancer series and is itself therefore not such a useful discriminator as peritoneal involvement. ${ }^{18}$ The importance of peritoneal involvement has been exemplified more recently by the institution of trials of intraperitoneal chemotherapy in Europe and North America, and the success of those trials. ${ }^{17}{ }^{30} 31$ This study has once again underpinned the importance of this pathologically derived parameter in prognosis and its potential utility in selecting patients for chemotherapy, whether systemic or intraperitoneal.

While mesorectal (deep, circumferential, radial, "lateral") margin involvement has been much studied in rectal cancer, ${ }^{14}{ }^{15}$ little attention has been paid to surgical margins in colonic cancer. Admittedly, most of the ascending, transverse, and descending colon is invested in peritoneum, and surgical margins are less important than in the lower rectum where the mesorectum is effectively circumferential. However, in the caecum, proximal ascending colon, and sigmoid colon, surgical margins, both retroperitoneal and mesocolic, are more relevant. Indeed, this series has shown that margin involvement has independent prognostic significance in Dukes' B colon cancer. Furthermore, it has shown that tumour can apparently seed across an inflammatory focus, present at a margin, to allow metastasis, subsequent relapse, and death, even if the tumour itself is not demonstrated at a margin, as long as there is continuity through the inflammation between the tumour and margin. This is most relevant in the sigmoid colon where the common coexistence of carcinoma and diverticular disease leads to tumorous obstruction of diverticula, secondary diverticulitis, and potential margin involvement through the inflammatory focus. From data in this study, the "inflamed margin" can be usefully combined with frank margin involvement to simplify the prognostic model.

The adverse prognosis of perforation through the tumour has been previously demonstrated. In this series of Dukes' B colon cancer, this adverse prognostic influence was seen only in the first postoperative year. Nevertheless, perforation remains a pathologically determined feature of extreme adverse prognostic significance and one that demands further studies into the efficacy of adjuvant chemotherapy, whether systemic and/or, seemingly more logically, intraperitoneal.

All four pathological parameters found to have independent prognostic significance in this study do not suffer the problems of subjectivity of other pathologically determined parameters, such as those forming part of the Jass classification. ${ }^{26}$ Nevertheless, their detection depends critically on accurate specimen dissection and block selection. In the past, the ability of diagnostic pathologists to provide such data has been suboptimal. ${ }^{32}$ There is now evidence that pathologists can at least record such data accurately, particularly with the advent of proforma reporting, such as those introduced by the Royal College of Surgeons, the Association of Coloproctology, UKCCCR, and the Royal College of Pathologists. ${ }^{33}$

The cumulative PI, in this series based on the four pathologically determined parameters, provides prognostic categories that could be used to guide the decision concerning adjuvant therapy. For Dukes' B colon cancer as a whole, the efficacy of chemotherapy remains controversial. ${ }^{4-12}$ Thirty one per cent of patients in this study had none of the four adverse independent prognostic factors and a five year survival rate of $94 \%$, effectively the same, in our series, as that of Dukes' A cancers. ${ }^{18}$ As adjuvant therapy is required to demonstrate a $5 \%$ increase in survival before it can be considered efficacious and cost effective, $^{719}$ it could not seemingly be justified in this patient group. On the other hand, our data enabled the categorisation of high risk patients with a survival rate of $49.8 \%$ that might well be improved by adjuvant therapy.

By combining a meticulous pathological technique with a restricted analysis of Dukes' B colonic cancer alone, we have been able to demonstrate that particular pathological factors can provide invaluable prognostic information in this group of patients, especially when part of a cumulative prognostic index. Our analysis made full use of the dataset with the internal validation corroborating the prognostic model. However, before initiating extensive use of this model for selecting patients for adjuvant therapy, it is important to confirm the model in independent datasets. After confirmation in such an external dataset, we would propose that the analysis of these pathological factors could form the basis for prospective controlled trials of adjuvant systemic and/or intraperitoneal chemotherapy in Dukes' B colonic cancer.

\section{ACKNOWLEDGEMENTS}

We are indebted to the Imperial Cancer Research Fund and Professor Nicholas A Wright for financial support for this and other Gloucester pathological studies. We gratefully acknowledge the help, advice, and statistical expertise of Dr Douglas G Altman. We would like to thank our Gloucester surgical colleagues, Mr W H F Thomson, Professor M W L Gear, Professor H Barr, Miss M E Lucarotti, Mr B P Heather, Mr J J Earnshaw, Mr J O Kilby, Mr D J Jones, and the late Mr D G Calvert, and the general practitioners of West Gloucestershire, who have ensured 
comprehensive surveillance of all patients in the Gloucester Colorectal Cancer studies. We would also like to thank the histopathology staff of Gloucestershire Royal Hospital for their cooperation in our studies.

\section{Authors' affiliations}

V C Petersen, K J Baxter, N A Shepherd, Department of Histopathology and Cranfield Postgraduate Medical School in Gloucestershire, Gloucestershire Royal Hospital, Great Western Road, Gloucester GL1 3NN, UK

S B Love, Medical Statistics Laboratory, Imperial Cancer Research Fund, Oxford, UK

\section{REFERENCES}

1 Dukes CE, Bussey HJR. The spread of rectal cancer and its effect on prognosis. Br J Cancer 1958;12:1016-23.

2 Moertel CG, Fleming TR, Macdonald JS, et al. Levamisole and fluorouracil for adjuvant therapy of resected colon carcinoma. N Engl J Med 1990;322:352-8.

3 Moertel CG, Fleming TR, Macdonald JS, et al. Fluorouracil and levamisole as effective adjuvant therapy after resection of stage III colon carcinoma. Ann Intern Med 1995;122:321-6.

4 International Multicentre Pooled Analysis of B2 Colon Cancer Trials (IMPACT) Investigators. Efficacy of adjuvant fluorouracil and folinic acid in colon cancer. Lancet 1995;345:939-44.

5 Dube S, Heyen F, Jenicek M. Adjuvant chemotherapy in colorectal carcinoma: results of a meta-analysis. Dis Colon Rectum 1997:40:35-41.

6 Norum J, Vonen B, Olsen JA, et al. Adjuvant chemotherapy (5-fluorouracil and levamisole) in Dukes' B and C colorectal carcinoma. A cost-effectiveness analysis. Ann Oncol 1997;8:65-70.

7 Zaniboni A, Labianca R, Marsoni S, et al. GIVIO-SITAC 01: A randomized trial of adjuvant 5-fluorouracil and folinic acid administered to patients with colon carcinoma-long term results and evaluation of the indicators of health-related quality of life. Cancer 1998;82:2135-44.

8 Wolmark N, Rockette H, Fisher B, et al. The benefit of leucovorin-modulated fluorouracil as postoperative adjuvant therapy for primary colon cancer: Results from National Surgical Adjuvant Breast and Bowel Project protocol C-03. J Clin Oncol 1993;11:1879-87.

9 Francini G, Petrioli R, Lorenzini L, et al. Folinic acid and 5-fluorouracil as adjuvant chemotherapy in colon cancer. Gastroenterology 1994;106:899-906.

10 Erlichman C, Marsoni S, Seitz JF, et al. Event free and overall survival is increased by FUFA in resected B colon cancer: A pooled analysis of five randomised trials (RCTS). Proc Am Soc Clin Oncol 1997; 16:991.

11 O'Connell MJ, Mailliard JA, Kahn M, et al. Controlled trial of fluorouracil and low-dose leucovorin given for six months as postoperative adjuvant therapy for colon cancer. J Clin Oncol 1997:15:246-50.

12 Mamounas E, Wieand S, Wolmark N, et al. Comparative efficacy of adjuvant chemotherapy in patients with Dukes' B versus Dukes' $C$ colon cancer: results from four national surgical adjuvant breast and bowel project adjuvant studies (C-01, C-02, C-03 and C-04). J Clin Oncol 1999; 17:1349-60.
13 International Multicentre Pooled Analysis of B2 Colon Cancer Trials (IMPACT B2) Investigators. Efficacy of adjuvant fluorouracil and folinic acid in B2 colon cancer. J Clin Oncol 1999;17:1356-68.

14 Quirke P, Dixon MF, Durdey P, et al. Local recurrence of rectal adenocarcinoma due to inadequate surgical resection. Histopathological study of lateral tumour spread and surgical excision. Lancet 1986:ii:996-9.

15 Adam IJ, Mohamdee MO, Martin IG, et al. Role of circumferential margin involvement in the local recurrence of rectal cancer. Lancet 1994;344:707-10.

16 Cawthorn SJ, Parums DV, Gibbs NM, et al. Extent of mesorectal spread and involvement of lateral resection margin as prognostic factors after surgery for rectal cancer. Lancet 1991;335:1055-9.

17 Scheithauer W, Kornek GV, Marczell A, et al. Combined intravenous and intraperitoneal chemotherapy with fluorouracil+leucovorin vs fluorouracil+levamisole for adjuvant therapy of resected colon carcinoma. Br J Cancer 1998;77:1349-54.

18 Shepherd NA, Baxter KJ, Love SB. The prognostic importance of peritoneal involvement in colonic cancer: a prospective evaluation. Gastroenterology 1997;112:1096-102.

19 Norum J. Adjuvant chemotherapy in Dukes' B and C colorectal carcinoma has only a minor influence on psychological distress. Support Care Cancer 1997;5:318-21

20 Talbot IC, Ritchie S, Leighton $\mathrm{MH}$, et al. The clinical significance of invasion of veins by rectal cancer. Br J Surg 1980;67:439-42.

21 Sasaki O, Atkin WS, Jass JR. Mucinous carcinoma of the rectum. Histopathology 1987:11:259-72.

22 Shepherd NA, Saraga EP, Love SB, et al. Prognostic factors in colonic cancer. Histopathology 1989;14:613-20

23 Jass JR, Atkin WS, Cuzick J, et al. The grading of rectal cancer: historical perspectives and a multivariate analysis of 447 cases Histopathology 1986;10:437-59.

24 Halvorsen TB, Seim E. Association between invasiveness, inflammatory reaction, desmoplasia and survival in colorectal cancer. J Clin Pathol 1989:42:162-6.

25 Shepherd NA, Baxter KJ, Love SB. Influence of local peritoneal involvement on local recurrence and prognosis in rectal cancer. J Clin Pathol 1995;48:849-55.

26 Jass JR, Love SB, Northover JMA. A new prognostic classification of rectal cancer. Lancet 1987;i:1303-6.

27 Parmar MKB, Machin D. Survival analysis: a practical approach. Chichester: John Wiley, 1995

28 Sauerbrei W, Schumacher M. A bootstrap resampling procedure for model building: application to the Cox regression model. Stat Med 1992;11:2093-109

29 Newland RC, Dent OF, Chapuis PH, et al. Survival after curative resection of lymph node negative colorectal carcinoma. Cancer 1995;76:564-71

30 Sugarbaker PH. Mechanisms of relapse for colorectal cancer: implications for intraperitoneal chemotherapy. J Surg Oncol Supp $1991 ; 2: 36-41$.

31 Vaillant JC, Nordlinger B, Deuffic S, et al. Adjuvant intraperitonea 5 -fluorouracil in high-risk colon cancer: A multicenter phase III trial. Ann Surg 2000;231:449-56.

32 Shepherd NA, Quirke P. Colorectal cancer reporting: are we failing the patient? J Clin Pathol 1997;50:266-7.

33 Cross SS, Feeley KM, Angel CA. The effect of four interventions on the informational content of histopathology reports of resected colorectal carcinomas. J Clin Pathol 1998;51:481-2. 